\title{
Design of an On-Axis Coupled Planar MM-Wave Structure
}

\author{
R. Apel and H. Henke \\ Technische Universität Berlin, EN-2 Einsteinufer 17, D-10587 Berlin, Germany
}

\begin{abstract}
The paper presents the design of an on-axis coupled structure which is planar double-sided and suited for fabrication with deep X-ray lithography. It is a standing wave structure with an operating frequency of $91.392 \mathrm{GHz}$ and is designed for the $\pi$-mode. End cells and input power coupler are matched numerically by means of the code GdfidL. The main RF parameters are also determined numerically. Reflection and bead pull measurements with a scaled-up model at $10 \mathrm{GHz}$ are presented.
\end{abstract}

\section{INTRODUCTION}

Very high frequency accelerating structures will explore new parameter ranges and new applications. Among other things, a very attractive feature and a potential breakthrough concept is an 'integrated' accelerator module, i.e. the manufacturing of accelerating structures, power sources and focusing elements with one technology and on a common support. The avenue to realize this vision is X-ray lithography (LIGA). It meets the requirements in fabricational precision, 1 micrometer tolerances and about $0.1 \mathrm{mi}-$ crometer surface roughness, and it allows for nearly arbitrarily complicated structures as long as they stay planar.

That is where the skill of the design engineer is challenged: to develop and build planar structures and components.

Based on the concept of a doubled-sided, open muffintin structure [1] we have been working on different devices [2]. Since we believe that a constant impedance structure is easier to build and that a standing-wave (SW) structure with equal power dissipation facilitates cooling and operation we had at first designed a side-coupled SW muffintin [3]. However, the RF parameters, especially bandwidth and attenuation, were not very satisfactory. Therefore in the present paper, we present an on-axis coupled doubleperiodic muffin-tin with a larger aperture, lower attenuation and large bandwidth.

\section{STRUCTURE GEOMETRY AND RF PARAMETERS}

Since the RF power dissipation increases with the square root of the frequency and since additional losses due to surface roughness are inevitable, high frequency structures have high losses. Therefore, an adequate cooling and temperature control system may impose a constant-gradient operation. But a constant-gradient structure is not a straight forward solution for a fully planar geometry. On the other hand, SW structures have a constant gradient but a lower shunt impedance. The only exception is zero- or $\pi$-mode operation, where the group velocity is small, the structure is sensitive to fabricational errors and only a few cells can be coupled. The apparent solution to this dead end are doubleperiodic structures which are confluent and have high shunt impedance and group velocities. The first solution we had was a side-coupled structure [3]. Although the paper design and a scaled-up model behave reasonably well, we were not completely satisfied with the group velocity and a distorted Brillouin diagram due to the direct coupling between main cells. Therefore, we started the design of an on-axis coupled structure, Figure 1.



Figure 1: Topview and cross section of an on-axis coupled muffin-tin: $g=0.980, w=2.231, t=0.230, w 1=$ $3.374, g 1=0.200,2 a=1.050,2 b=2.540$ (in mm)

The iris thickness and the gap of the coupling cells were fixed to $t=0.23 \mathrm{~mm}$ and $g 1=0.20 \mathrm{~mm}$, respectively. The period length follows from the $\pi$-mode operation as

$$
L=\pi / \beta=\lambda / 2=1.64 \mathrm{~mm} .
$$

The beam aperture is in principle a free parameter and influences bandwidth, shunt impedance and wakefields. As a trade-off we chose

$$
2 a / \lambda=0.32 \Rightarrow 2 a=1.05 \mathrm{~mm} .
$$

The other dimensions were calculated with GdfidL [4] and are shown in Figure 1. The resulting RF parameters are given in Table 1. As a comparison the Table shows also the parameters of the side-coupled structure (SCS) and of a single-periodic muffin-tin (SPMT). As can be seen, the onaxis coupled structure (OACS) has the largest bandwidth, highest group velocity and lowest attenuation. An estimate of the number of cells which can be coupled together gives

$$
\frac{\omega}{Q_{0}}<\text { mode spacing }=\frac{\pi}{4} \frac{B}{N} \text { or } N<136 .
$$




\begin{tabular}{|c|c|c|c|c|}
\hline Symbol & OACS & SCS & SPMT & Unit \\
\hline$f$ & 91.392 & 91.392 & 91.392 & $G H z$ \\
$r / Q_{0}$ & 58 & 53.8 & 81.6 & $k \Omega / m$ \\
$r$ & 155 & 166 & 200 & $M \Omega / m$ \\
$Q_{0}$ & 2680 & 3090 & 2490 & \\
$\alpha$ & 3.6 & 8.6 & 4.1 & $1 / m$ \\
$v_{g} / c_{0}$ & 0.10 & 0.036 & 0.094 & \\
$B$ & 11.9 & 7.8 & 9.3 & $G H z$ \\
$B^{\prime}$ & 0.13 & 0.085 & 0.102 & \\
\hline
\end{tabular}

Table 1: RF parameters for $2 a=1.05 \mathrm{~mm}$

Then assuming a central feeding point and an attenuation parameter $\tau=\alpha l=0.18$ an overall structure length $l$ of $10 \mathrm{~cm}$ comprising 60 main cells seems to be a good design goal. The large bandwidth and the resulting high group velocity together with the point of confluence can be seen in the beautifully symmetric Brillouin diagram, Figure 2.

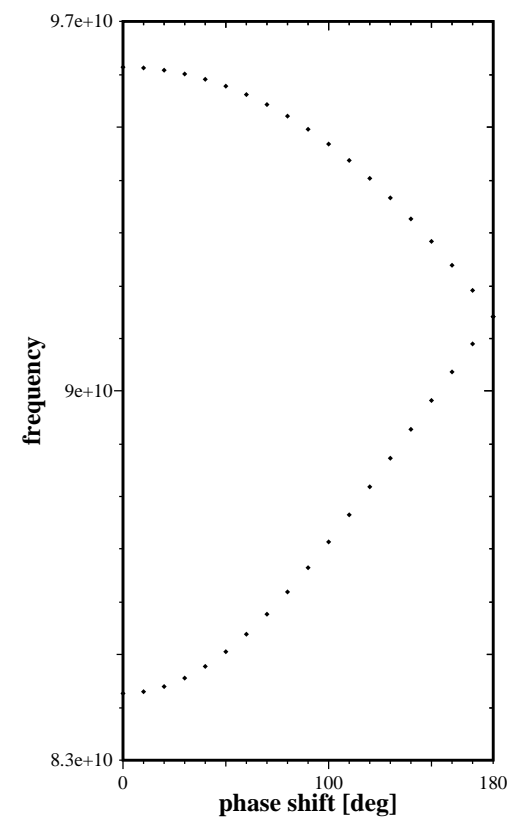

Figure 2: Brillouin diagram for OACS

\section{FULL STRUCTURE WITH END CELLS AND COUPLER}

Special care has to be taken to get a flat $\pi$-mode distribution. This requires a detuning of the end cells which was done with $G d f i d L$ and is shown in Figure 3.

For the power coupler we have chosen a straight forward solution for the time being. The feeding line couples to the mid-cell via the side-wall, Figure 3 . Since we did not want a cut in the coupling iris for fabricational reasons and the coupling was undercritical we added a double $\lambda / 4$ stub-line for the match. We also had to add a $\lambda / 4$ transmission-line transformer and special matching elements in the side-wall in order to taper and match the groove-guide to the rectan- gular guide. The resulting match of the whole structure was found with the time-domain option of $G d f i d L$ and is given in Figure 4. The losses were simulated by a lossy dielectric filling.

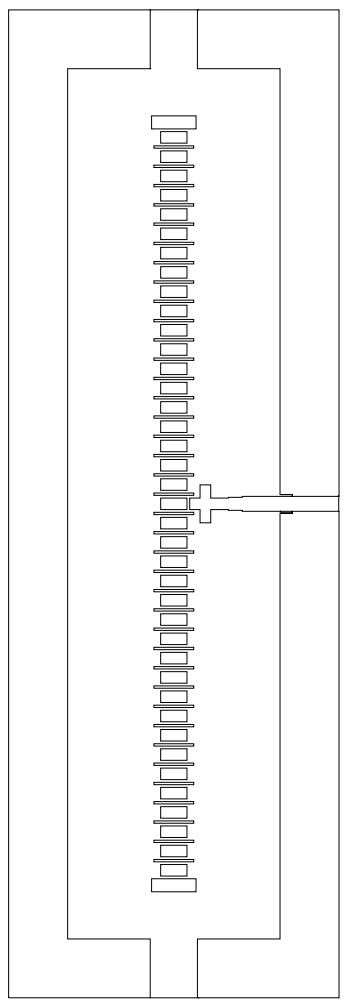

Figure 3: Full structure with feeding line, coupler and detuned end cells

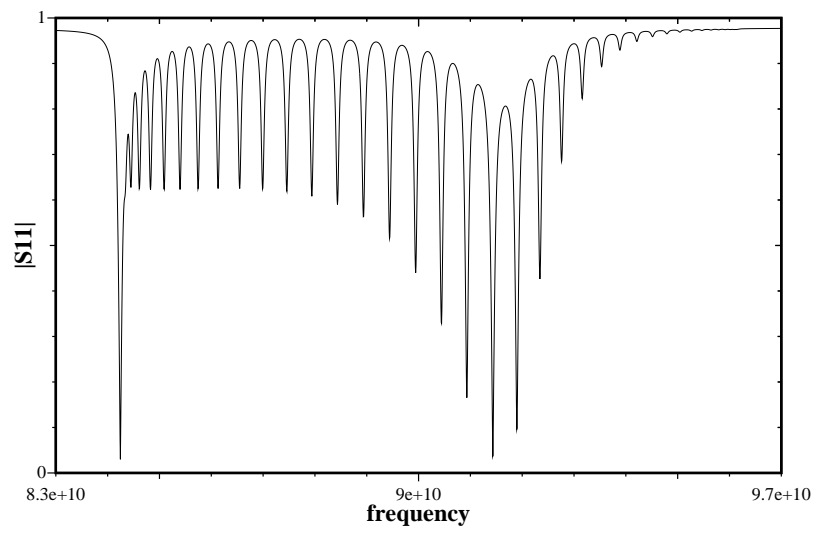

Figure 4: Simulated magnitude of $S_{11}$ of the full structure.

\section{SCALED-UP MODEL AND PROTOTYPE}

In order to verify the design we build an Al-model scaled to 10.2 GHz with seven main cells, see Figure 5. Reflection measurement and bead pull measurement of the $\pi$-mode were done with a network analyzer (HP 8722C), Figures 6 


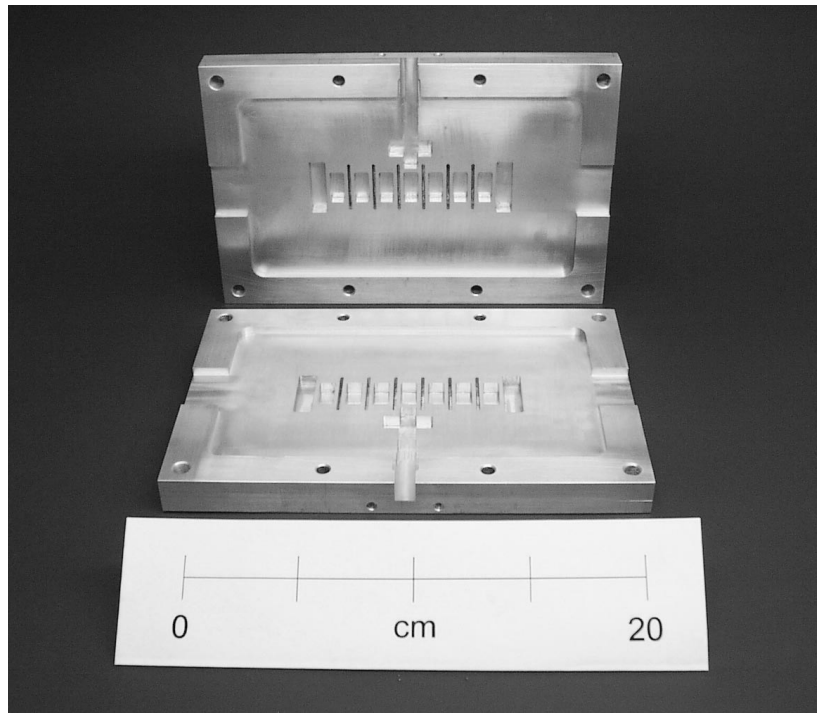

Figure 5: Scaled-up model

and 7. The measured frequency response is in principal in good agreement with the simulation. Only one thing still requires improvement. If we compare the $G d f i d L$ simulation to the measured reflection coefficient, we have only four measured resonances instead of six resonances in the simulation, see Figure 6. One reason for this may be tolerances during the fabricational process.



Figure 6: Reflection coefficient for the scaled-up model

Finally, bead pull measurement of the $\pi$-mode has been performed. The bead was made by silver painting on a pure cotton fiber. Figure 7 shows the reflection coefficient of the $\pi$-mode in resonant condition. One can see the seven main cells with resonant fields and a clear $\pi$-mode field distribution along the beam axis. Since the feeding line couples to the mid-cell, the field distribution is nearly symmetrical w. r. t. this cell and the field decays slightly to the structure ends. Due to the good results of the scaled-up model we have built a first LIGA mask for a $91 \mathrm{GHz}$ structure with 39 main cells, the end cells, the coupler and the transformers. The department of precision and micro technology at the TU-Berlin has started to build up the electroplating.

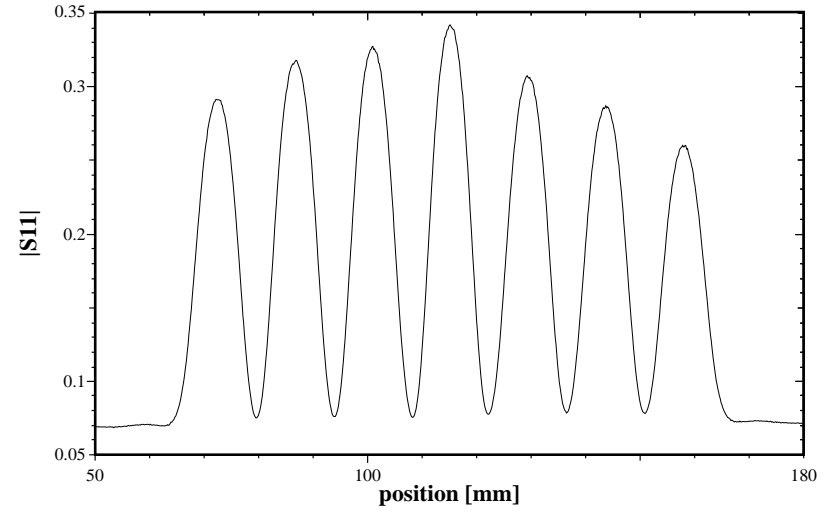

Figure 7: Bead pull measurement for the scaled-up model

\section{CONCLUSION}

The concept of an on-axis coupled, double-periodic muffintin shows very attractive features for a very high frequency SW-structure. It has an exceptional wide bandwidth and high group velocity and is therefore insensitive to fabricational errors. The shunt impedance is reduced only about $25 \%$ as compared to the single-periodic, $2 \pi / 3$-mode structure. The relatively low attenuation allows for coupling a large number of cells. About 60 cells should be possible and would give a $10 \mathrm{~cm}$ long structure at $91 \mathrm{GHz}$. This corresponds just to a 4 inch wafer for a LIGA mask and yields a sound attenuation parameter $\alpha l=0.18$. A model scaled-up to $10.2 \mathrm{GHz}$ has been manufactured. Measurements have verified the design parameters well. Therefore a first LIGA mask for a $91 \mathrm{GHz}$ structure with 39 main cells has been made and electroplating of the structure is under process.

\section{ACKNOWLEDGEMENT}

All the LIGA related work on the prototype is and will be in collaboration with the department of precision and micro technology at the TU-Berlin, H. Lehr and M. Schmidt.

\section{REFERENCES}

[1] H. Henke, Y.W. Kang and R.L. Kustom, "A mm-wave RF Structure for Relativistic Electron Acceleration", Argonne National Laboratory, internal report ANL/APS/MMW-1, 1993.

[2] H. Henke, R. Merte, A. Nassiri, J. Song, Y.W. Kang and R.L. Kustom, "Millimeter-Wave RF Structure", Proceedings of the XVIII International Linear Accelerator Conference, CERN, Geneva, internal report 96-07, 15 Nov. 1996, Vol. II, pp524.

[3] W. Bruns, H. Henke and R. Merte, "Design of a $94 \mathrm{GHz}$ Accelerating Structure", Proceedings of the 5th European Accelerator Conference, Barcelona, June 1996.

[4] W. Bruns, "GdfidL: A finite difference program for arbitrarily small perturbations in rectangular geometries", IEEE Trans. Magn. Vol. 32, No. 3 May 1996. 